\title{
Factors influencing postmenopausal women's quality of sleep
}

\author{
Thaís Gigliotti Malheiros Luzo*, Bárbara Valença Pereira Conde', James Kageyama Coelho', José Mendes Aldrighi \\ ${ }^{1}$ Faculdade de Ciências Médicas da Santa Casa de São Paulo, São Paulo, SP, Brasil
}

\begin{abstract}
Objective: Verify the factors associated with poor sleep quality among postmenopausal women. Methods: Cross-sectional, descriptive, observational study conducted with 185 postmenopausal women assisted at the outpatient clinic of Santa Casa de Sao Paulo. Data were collected regarding sleep quality using the Pittsburgh Sleep Quality Index (PSQI); quality of life according to the WHO Quality of Life-BREF (WHOQOL-BREF) and the Alcohol Use Disorders Identification Test (AUDIT); and through the application of a questionnaire including information on socioeconomic profile, age of menopause, natural or surgical menopause, smoking, chronic diseases, use of hormonal therapy, hot flashes, and alcohol consumption. Data were analyzed using STATA 13 statistical software. Variables were compared by mean and standard deviation. Regression analysis was used to verify the possible factors associated with quality of sleep. The coefficients are considered significant when $p<0.10$. Results: $57.28 \%$ of the women investigated presented poor sleep or sleep disturbance. Negative correlation was observed between quality of sleep and smoking $(p=0.083)$, whereas positive correlation was found between poor sleep quality and number of hot flashes $(p=0.00)$. In addition, the earlier the menopause, the worse the quality of sleep $(p=0.05)$. Conclusions: The following factors negatively influencing postmenopausal women's quality of sleep were identified: smoking, number of hot flashes, and age of menopause. It is not possible to identify causality; however, worsening sleep quality was identified when these factors were present.
\end{abstract}

Keywords: post menopausal period; sleep; smoking.

\section{Introduction}

Hormonal changes caused by women's ovarian failure during the climacteric cause symptoms such as hot flashes, fatigue, depression, and irritability, which when associated with other factors can alter their quality of sleep, affecting their work, personal relationships, and well-being. ${ }^{1,2}$

This study focuses on sleep quality in postmenopausal women. Justification is based on the fact that 40 to $60 \%$ of women report sleep disturbance during menopause, and the etiology of this impairment is probably multifactorial. ${ }^{1}$ The Pittsburgh Sleep Quality Index (PSQI), which has been cited in more than 1700 scientific papers at PubMed database, is frequently used by the international scientific community to evaluate quality of sleep subjectively; whereas polysomnography and electroencephalography ${ }^{3}$ are used in objective sleep quality assessment. Several studies using these methods indicate that poor sleep quality increases with age, and that insomnia is a prevalent complaint among postmenopausal women., ${ }^{3,4}$ A study conducted in China between July 2012 and May 2013 with 2429 women aged 40-59 years compared the scores on the PSQI between the different stages of menopausal status and showed significantly higher scores at postmenopause, revealing poorer quality of sleep. ${ }^{3}$ The study demonstrated that marital status, family income, and regular physical exercise are also correlated with sleep quality. ${ }^{3}$ Sleep disorders may be associated with increased prevalence of obesity and arterial hypertension, decreased health, increased mortality risk, hormonal and biochemical changes, increase in health care expenditures, and increased risk of psychological diseases, especially depression.5,6

Financial support: None.

Conflicts of interest: The authors declare no conflicts of interest.

Submitted: January 03, 2018.

Accepted: February 23, 2018.

The study was carried out at Hospital Santa Casa de São Paulo (HSCSP) - SP, Brazil.

Copyright Luzo et al. This is an Open Access article distributed under the terms of the Creative Commons Attribution License, which permits unrestricted use, distribution, and reproduction in any medium, provided the original work is properly cited. 
With the increase in life expectancy in Brazil, women also spend a longer time in postmenopause, and they need to cope with the changes caused by hormonal decline for longer. ${ }^{3}$ Although there are many studies on the theme of sleep quality in the literature, only a few of them address this period, which has become increasingly long in women's lives. Therefore, this study aims to identify the possible factors that influence the quality of sleep in this population so that interventions to promote a healthier life with fewer diseases and comorbidities can be discussed.

\section{Objective}

Verify the factors associated with poor sleep quality among postmenopausal women.

\section{Methods}

After approval by the Research Ethics Committee of the College of Medical Sciences of Santa Casa de Sao Paulo, the study was conducted with 185 postmenopausal women assisted at the various medical specialties of the Conde de Lara outpatient clinic in the Santa Casa de Sao Paulo hospital complex between September and December 2016.

This observational, cross-sectional, descriptive study was conducted using the following instruments: Pittsburgh Sleep Quality Index (PSQI) to investigate quality of sleep; WHO Quality of Life-BREF (WHOQOL-BREF) to evaluate quality of life; Alcohol Use Disorders Identification Test (AUDIT) to assess alcohol consumption; anamnesis prepared by the researchers. The PSQI instrument classifies sleep as good, poor, and sleep disturbance; it is subdivided into seven domains: subjective sleep quality, sleep latency, sleep duration, sleep efficiency, daytime dysfunction, sleep fragmentation, and use of sleep aid medications during the past month. The WHOQOL-BREF instrument comprises 24 items that measure the following domains: physical health, psychological health, social relationships, and environment. Results are given in percentage for each of the domains, as well as for global quality of life, which is a mean score of all domains scaled in a positive direction - the closer the index is to 100\%, the better. The AUDIT instrument is composed of 10 questions that, according to the answer, result in a score separating the following groups: Zone I, low risk consumption or abstinence; zone II, risky consumption; zone III, harmful consumption or even dependence; zone IV, dependence. In addition to the variables of interest, the questionnaire also addressed questions on socioeconomic conditions, age of menopause, natural or surgical menopause, smoking (smoker, non-smoker, or former smoker), chronic diseases, use of hormone therapy, hot flashes quantifying how many a day and a week, and intensity of alcohol intake. Data were analyzed using STATA 13 statistical software. Variables were compared by mean and standard deviation. Linear regression analysis was used to verify the possible factors associated with quality of sleep. The coefficients are considered significant when $p<0.10(90 \%$ statistical significance level).

\section{Results}

Study participants were 185 women with mean age of 61.2 years $(S D=8.0)$ and mean age of menopause of 46.6 years $(S D=6.7)$. Their average family monthly income was 1.1 Brazilian minimum wages $(S D=1.5)$, with $91.9 \%$ of them receiving up to two minimum wages and only $1.6 \%$ receiving between 5 and 12 minimum wages. With respect to schooling, $89.7 \%$ had never been to school or had incomplete High School and $8.1 \%$ had College degrees.

Most of the study sample (57.28\%) was composed of women who presented poor sleep or sleep disturbance. The results showed that $62.15 \%$ of the interviewees presented hot flashes and $38.91 \%$ were former smokers or smokers, and that these factors possibly influenced their quality of sleep negatively (Table 1).

Analysis of these factors shows that smoking worsens the quality of sleep ( $p=0.083)$, with $62.5 \%$ of the former smoking or smoking women presenting poor sleep or sleep disturbance. Hot flashes were also negatively correlated with poor sleep quality $(p=0.00)$, with $71.21 \%$ of the women who reported having hot flashes also presented poor sleep or sleep disturbance. Age of menopause also interfered with sleep quality: the younger the age, the worse the sleep $(p=0.05)$ (Table 2).

\section{Discussion}

Postmenopause begins one year after the final menstrual period, which occurs around the age of 50 - it is considered early if it occurs before the age of 40 and late if it occurs after the age of 52 - and causes a series of hormonal changes in women, affecting their routine. ${ }^{1}$ These alterations occur because of ovarian failure owing to a gradual decrease of the primordial follicles over the years, which causes a decrease in progesterone and, mainly, in estradiol $[1,8]$. As a consequence, the pituitary hormones LH and FSH have no inhibitory stimulus, thus presenting increased blood concentration. ${ }^{1,6}$ Quality of sleep is one of the factors that influence the routine of these women due to these hormonal changes. Sleep disorders involve both health issues - such as lack of energy, constant headaches, stomachaches, risk of mental disorders, and 
Table 1. Profile of study participants

w

Total number of participants (185)

Age (years) $M+-S D$

Monthly income (number of Brazilian minimum wages) M +- DP

Schooling

1. Never been to school

2. Elementary School (incomplete)

3. Elementary School (complete)

4. High School (incomplete)

5. High School (complete)

6. College (incomplete)

7. College Degree

Age of menopause (years) M +- DP

Menopausal status

Natural

Surgical

Presence of hot flashes

Alcohol consumption

Quality of sleep

Good

Poor

Disturbance

Smoking

Neck circumference $(>37 \mathrm{~cm})$

(Of 148 participants)

Quality of life M +- DP

SAH

Diabetes Mellitus

Hypercholesterolemia

Hypothyroidism

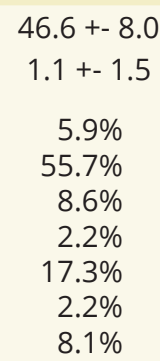

$46.6+-8.0$

$1.1+-1.5$

$5.9 \%$

$55.7 \%$

$8.6 \%$

$2.2 \%$

$17.3 \%$

$2.2 \%$

$8.1 \%$

$46.6+-6.7$

$74.6 \%$

$25.4 \%$

$62.16 \%$

$40 \%$

$42.72 \%$

$39.45 \%$

$17.83 \%$

$38.91 \%$

$29.1 \%$

$67.4+-13.7$

$58.4 \%$

$21.6 \%$

$16.8 \%$

$9.7 \%$

Captions: $\mathrm{M}$ = mean; SD = standard deviation; SAH = systemic arterial hypertension.

Table 2. Results associated with the variable quality of sleep

\begin{tabular}{|c|c|c|c|}
\hline Explanatory variables & Coefficient & \multicolumn{2}{|c|}{ [95\% Confidence Interval] } \\
\hline Smoking a & -0.15056 & -0.31036 & 0.00924 \\
\hline Neck circumference & 0.02967 & -0.18573 & 0.24507 \\
\hline Alcohol consumption & 0.01408 & -0.11138 & 0.13955 \\
\hline Menopausal status & 0.03747 & -0.20949 & 0.28442 \\
\hline Age & 0.00824 & -0.00518 & 0.02167 \\
\hline Decreased alcohol consumption & 0.01348 & -0.07541 & 0.10237 \\
\hline Late menopause & 0.26592 & -0.11384 & 0.64569 \\
\hline Age of menopause ${ }^{b}$ & 0.17119 & 0.00129 & 0.03294 \\
\hline Hot flashes (total) ${ }^{c}$ & -0.00578 & -0.00871 & -0.00286 \\
\hline $\mathrm{SAH}$ & 0.13672 & -0.08051 & 0.35396 \\
\hline DM & -0.09397 & -0.35482 & 0.16689 \\
\hline Hypercholesterolemia & 0.12757 & -0.15975 & 0.41488 \\
\hline Hypothyroidism & 0.21690 & -0.14456 & 0.57836 \\
\hline Intervention targeting SAH and DM & -0.08134 & -0.36197 & 0.19929 \\
\hline
\end{tabular}

Captions: $\mathrm{DM}=$ Diabetes mellitus; $\mathrm{SAH}=$ systemic arterial hypertension. Note: ${ }^{\mathrm{a}} p<10 \%$; ${ }^{\mathrm{b}} p<5 \%$. and $\mathrm{c} p<1 \%$. 
economic issues - such as increased hospitalization and risk of traffic accidents. ${ }^{7}$ Therefore, understanding the factors that may be associated with sleep disturbance is essential to treat, prevent, or ameliorate this condition.

Poor quality of sleep affects $30-70 \%$ of the adult population. Among postmenopausal women, this value varies from 40 to $60 \%$, and it is $48 \%$ in perimenopause according to the study by Kloss et al. ${ }^{2,3}$ In the present study, the PSQI was used to assess sleep quality, with sensitivity and specificity of 98.3 and $90.2 \%$, respectively, according to Zhang et al. ${ }^{1}$ Our results show that $57.28 \%$ of menopausal women presented poor sleep or sleep disturbance - values within the means for adults and climacteric, and above that for perimenopause. Considering this prevalence, the importance of this symptom is closely monitored in women during this period of life. Although it is believed that quality of sleep presents multifactorial influence, it is known that variation in the levels of gonadal hormones is one of its causes. Therefore, variation of estrogens during menopausal transition enables sleep disturbance. ${ }^{8}$ Thus, in addition to finding results similar to those of previous researches, this study sought to evaluate other causes of this multifactorial disorder; it investigated the influence of hot flashes, smoking, and age of menopause.

Prevalence of hot flashes is approximately $41.5 \%$ during perimenopause. ${ }^{9}$ In this study, assessment of this postmenopausal symptom revealed prevalence of $62.15 \%$, which suggests that hot flashes are more frequent after menopause. Because most of the participants present this symptom, we investigated whether it affected sleep quality, and the results obtained showed that $71.21 \%$ of these women present poor sleep or sleep disturbance. Statistical analysis identified positive correlation between worsening of sleep quality and increased number of hot flashes per day and per week. This suggests that the number of hot flashes is one of the factors influencing sleep quality, as it has been demonstrated in a previous study. ${ }^{9}$

Smoking is also known as a high prevalence factor for insomnia in the general population; however, few studies have been conducted on the influence of this addiction in the life of menopausal women. ${ }^{10,11}$ This study confirmed the negative influence of smoking on sleep quality during this period.

In addition to smoking and hot flashes, positive correlation was also found between poor sleep quality and younger age of menopause in this study. Age of menopause ranged from 25 to 68 years, considering both natural and surgical menopause. This suggests that women who have had menopause at a younger age are more likely to present poor quality of sleep compared with women who have had menopause at an older age. However, it was not possible to draw conclusions about women at late menopause (over 55 years of age) because of the small number of participants with this characteristic, which presented no statistical significance.

Limitations to this study are associated with the fact that the sample presented very similar socioeconomic profile. Therefore, it is assumed that there is a bias of the participants' social status, considering that most of them had low income and little schooling and that the interviews were conducted at a public hospital outpatient clinic.

\section{Conclusions}

Quality of sleep is influenced by several factors: social, demographic, marital status, education level, alcohol consumption, smoking, age, and comorbidities, hindering assessment of only one of them. ${ }^{12,13}$ This study identified the following factors negatively influencing postmenopausal women's quality of sleep: smoking, number of hot flashes, and age of menopause. However, it is not possible to identify causality, because this was a cross-sectional study, not interventionist. Therefore, further studies need to be conducted on the theme in order to confirm the causality of these possible factors - given the prevalence of this disorder in women in this period of life, and to prevent, treat, or minimize this frequent complaint, promoting better quality of sleep and, consequently, better quality of life for menopausal women.

\section{References}

1. Zhang J-P, Wang YQ, Yan MQ, Li ZA, Du XP, Wu XQ. Menopausal symptoms and sleep quality during menopausal transition and postmenopause. Chin Med J. 2016;129(7):771-7. http://dx.doi.org/10.4103/0366-6999.178961. PMid:26996470.

2. Lampio L, Saaresranta T, Engblom J, Polo O, Polo-Kantola P. Predictors of sleep disturbance in menopausal transition. Maturitas. 2016;94:137-42. http://dx.doi.org/10.1016/j.maturitas.2016.10.004. PMid:27823734.

3. Kloss JD, Tweedy K, Gilrain K. Psychological factors associated with sleep disturbance among perimenopausal women. Behav Sleep Med. 2004;2(4):177-90. http://dx.doi.org/10.1207/s15402010bsm0204_1. PMid:15600054.

4. Madrid-Valero JJ, Martínez-Selva JM, Ribeiro do Couto B, Sánchez-Romera JF, Ordoñana JR. Age and gender effects on the prevalence of poor sleep quality in the adult population. Gac Sanit. 2017;31(1):18-22. http://dx.doi.org/10.1016/j.gaceta.2016.05.013. PMid:27474487.

5. Knutson KL. Sleep duration and cardiometabolic risk: a review of the epidemiologic evidence. Best Pract Res Clin Endocrinol Metab. 2010;24(5):731-43. http://dx.doi.org/10.1016/j.beem.2010.07.001. PMid:21112022. 
6. Attarian HP, Viola-Saltzman M. Sleep disorders in women: a guide to practical management. 2nd ed. Totowa: Humana Press; 2006. http://dx.doi.org/10.1007/978-1-59745-115-4.

7. Müller MR, Guimarães SS. Impacto dos transtornos do sono sobre o funcionamento diário e a qualidade de vida. Estud Psicol. 2007;24(4):519-28.

8. Soares CN. Insônia na menopausa e perimenopausa: características clínicas e opções terapêuticas. Arch Clin Psychiatry. 2006;33(2):103-9. http://dx.doi.org/10.1590/S0101-60832006000200010.

9. Fisher TE, Chervenak JL. Lifestyle alterations for the amelioration of hot flashes. Maturitas. 2012;71(3):217-20. http://dx.doi. org/10.1016/j.maturitas.2011.12.006. PMid:22285470.

10. Strine TW, Chapman DP. Associations of frequent sleep insufficiency with health-related quality of life and health behaviors. Sleep Med. 2005;6(1):23-7. http://dx.doi.org/10.1016/j.sleep.2004.06.003. PMid:15680291.

11. Cray LA, Woods NF, Herting JR, Mitchell ES. Symptom clusters during the late reproductive stage through the early postmenopause: observations from the Seattle midlife women's health study. Menopause. 2012;19(8):864-9. http://dx.doi.org/10.1097/ gme.0b013e31824790a6. PMid:22643229.

12. Tang J, Liao Y, Kelly BC, Xie L, Xiang YT, Qi C, et al. Gender and regional differences in sleep quality and insomnia: a general population-based study in Hunan Province of China. Sci Rep. 2017;7:7. PMid:28262807.

13. Grandner MA, Smith TE, Jackson N, Jackson T, Burgard S, Branas C. Geographic distribution of insufficient sleep across the United States: a county-level hotspot analysis. Sleep Health. 2015;1(3):158-65. http://dx.doi.org/10.1016/j.sleh.2015.06.003. PMid:26989761.

\section{*Correspondence}

Thaís Gigliotti Malheiros Luzo

Faculdade de Ciências Médicas da Santa Casa de São Paulo

Rua tejo, 99 - Bairro Jardim Luzitânia

CEP 04031-040, São Paulo, SP, Brasil

Tel.: +55 (11) 94101-2828

E-mail: tata_luzo@hotmail.com

\section{Authors information}

TGML- Medical student, Gynecology and Obstetrics Department, Santa Casa de Misericórdia de São Paulo Institution. BVPC- Medical student, Gynecology and Obstetrics Department, Santa Casa de Misericórdia de São Paulo Institution.

JKC- MD, Gynecology and Obstetrics Department, Santa Casa de Misericórdia de São Paulo Institution.

JMA- PhD, Gynecology and Obstetrics Department, Santa Casa de Misericórdia de São Paulo Institution.

\section{Authors contribution}

JMA and JKC were responsible for general supervision of the research group and review of the text. TGML and BVPC were responsible for research, discussion and writing of the text. 